Western University

Scholarship@Western

Brain and Mind Institute Researchers'

Publications

Brain and Mind Institute

9-1-2014

\title{
Sharp emergence of feature-selective sustained activity along the dorsal visual pathway.
}

Diego Mendoza-Halliday

Cognitive Neurophysiology Laboratory, Department of Physiology, McGill University, Montreal, Canada

Santiago Torres

Cognitive Neurophysiology Laboratory, Department of Physiology, McGill University, Montreal, Canada

Julio C Martinez-Trujillo

Cognitive Neurophysiology Laboratory, Department of Physiology, McGill University, Montreal, Canada

Follow this and additional works at: https://ir.lib.uwo.ca/brainpub

Part of the Neurosciences Commons, and the Psychology Commons

\section{Citation of this paper:}

Mendoza-Halliday, Diego; Torres, Santiago; and Martinez-Trujillo, Julio C, "Sharp emergence of featureselective sustained activity along the dorsal visual pathway." (2014). Brain and Mind Institute Researchers' Publications. 270.

https://ir.lib.uwo.ca/brainpub/270 


\title{
Sharp emergence of feature-selective sustained activity along the dorsal visual pathway
}

\author{
Diego Mendoza-Halliday, Santiago Torres \& Julio C Martinez-Trujillo
}

Sustained activity encoding visual working memory representations has been observed in several cortical areas of primates. Where along the visual pathways this activity emerges remains unknown. Here we show in macaques that sustained spiking activity encoding memorized visual motion directions is absent in direction-selective neurons in early visual area middle temporal (MT). However, it is robustly present immediately downstream, in multimodal association area medial superior temporal (MST), as well as and in the lateral prefrontal cortex (LPFC). This sharp emergence of sustained activity along the dorsal visual pathway suggests a functional boundary between early visual areas, which encode sensory inputs, and downstream association areas, which additionally encode mnemonic representations. Moreover, local field potential oscillations in MT encoded the memorized directions and, in the low frequencies, were phase-coherent with LPFC spikes. This suggests that LPFC sustained activity modulates synaptic activity in MT, a putative top-down mechanism by which memory signals influence stimulus processing in early visual cortex.

Humans and other primates can temporarily encode, store and keep within the focus of attention visual information unavailable to the eyes. This ability, known as working memory, allows past experiences to influence our current thoughts and behaviors, and is crucial to cognitive processes such as abstract thinking, decision-making and action planning ${ }^{1}$. During behavioral tasks that require macaque monkeys to temporarily memorize the locations or features of visual objects, such as a delayed match-to-sample task, neurons in highorder association areas such as the LPFC ${ }^{2,3}$, posterior parietal corex ${ }^{4}$ and inferotemporal cortex ${ }^{5}$ show elevated and sustained spiking activity that encodes the memorized information. This sustained activity in the absence of visual stimulation is considered by many as the neural correlate of working memory ${ }^{2-6}$.

It has been recently proposed that because neurons in high-order association areas may lack fine selectivity for single visual features (for example, direction of motion, color or orientation), working memory representations of such features must be encoded by feature-selective neurons in the early visual cortex ${ }^{7,8}$. However, electrophysiological studies in monkeys have reported contradictory evidence. During the delay period of a delayed match-to-sample task and similar tasks, the activity of early visual neurons has been found to be weak and transient ${ }^{9-11}$, absent ${ }^{12}$ or observed while visual stimulation remains inside the neurons' receptive field ${ }^{13}$. A puzzling finding in functional magnetic resonance imaging studies in humans is that during the same tasks, blood oxygenation level-dependent (BOLD) signals in early visual areas remain at baseline levels, yet the memorized features can be decoded from these signals using pattern-classification analysis techniques ${ }^{8,14,15}$. Currently, it remains highly controversial whether sustained activity encoding memorized visual features is present in early visual areas or whether it emerges further downstream along the visual pathways.
To examine this issue, we trained monkeys in a delayed match-tosample task that required memorizing the motion direction of a visual stimulus and recorded the activity of direction-selective neurons in three serially connected areas along the dorsal visual pathway: MT, an early visual area involved in motion processing ${ }^{16}$; its immediate downstream neighbor, $\mathrm{MST}^{17,18}$, a multimodal association area that integrates visual motion signals from MT with vestibular inputs ${ }^{19}$; and LPFC, a high-order association area further downstream that has been classically associated with coding of working memory ${ }^{3}$ (Fig. 1). We found that sustained spiking activity encoding the memorized motion direction was absent in MT neurons but was robustly present in MST and LPFC.

\section{RESULTS}

Behavioral task

We trained two macaque monkeys, $\mathrm{M}$ and $\mathrm{S}$, to perform a delayed match-to-sample task (Fig. 2a). In each trial, a sample random dot stimulus with motion in one of four directions was presented for $1 \mathrm{~s}$. After a variable delay period (1.2-2 s), two test stimuli were sequentially presented for $0.59 \mathrm{~s}$, one of which matched the direction of the sample. To obtain a juice reward, the monkeys had to release a button when the matching test appeared. We presented a behaviorally irrelevant stimulus, which served experimental purposes unrelated to the results reported here, in the opposite hemifield simultaneously with the tests. Because the location of the tests and irrelevant stimulus were randomly swapped from trial to trial, the monkeys could not reliably predict the location of the tests. The task required the monkeys to memorize the sample direction throughout the delay period. In all experimental sessions, the performance of both monkeys was well above the $50 \%$ chance level (mean of $79 \%$ for monkey $\mathrm{M}$ and $76 \%$ for monkey S). 
Figure 1 Anatomical location of recorded neurons. (a) Cortical surface showing LPFC in red (left), and locations of LPFC recording sites with respect to arcuate sulcus (AS) and principal sulcus (PS) (right). (b) Coronal MRI section showing MT in green and MST in blue (left). MRI section parallel to electrode trajectories (yellow and white lines) for each monkey, showing the location of all recorded neurons in MT and MST projected onto that section (right). Insets, magnification of the recorded regions, with black lines showing gray-white matter boundaries. STS, superior temporal sulcus.

\section{Sustained activity is present in MST and LPFC but not MT}

We recorded the responses of 631 neurons in the two monkeys: 112 neurons in MT (57 in monkey M and 55 in monkey S), 247 in MST (145 in M and 102 in S) and 272 in LPFC (118 in M and 154 in S). We identified MT and MST neurons by their motion direction tuning properties, anatomical location, and receptive field size and position (Fig. 1b and Supplementary Fig. 1). We recorded LPFC neurons from the cortical surface around the posterior end of the principal sulcus corresponding to Brodmann's areas 8 and 46 (ref. 20; Fig. 1a).

We first examined whether the sample direction was encoded by individual neurons in each of the three areas. We considered a neuron to be motion direction-selective if its firing rate significantly varied as a function of the sample direction (two-factor analysis of variance (ANOVA); Online Methods) during the sample period (sensory selectivity) or the delay period, while the animals memorized the sample direction (delay selectivity). We found that neurons in MT were sensory-selective but not delay-selective (Fig. 2b,c). To our surprise, immediately downstream from MT, in MST, we found neurons with both sensory and delay selectivity (Fig. 2d,e). In LPFC, neurons also showed both types of selectivity (Fig. 2f,g). Several MST and LPFC neurons showed strong delay selectivity but weak or no sensory selectivity

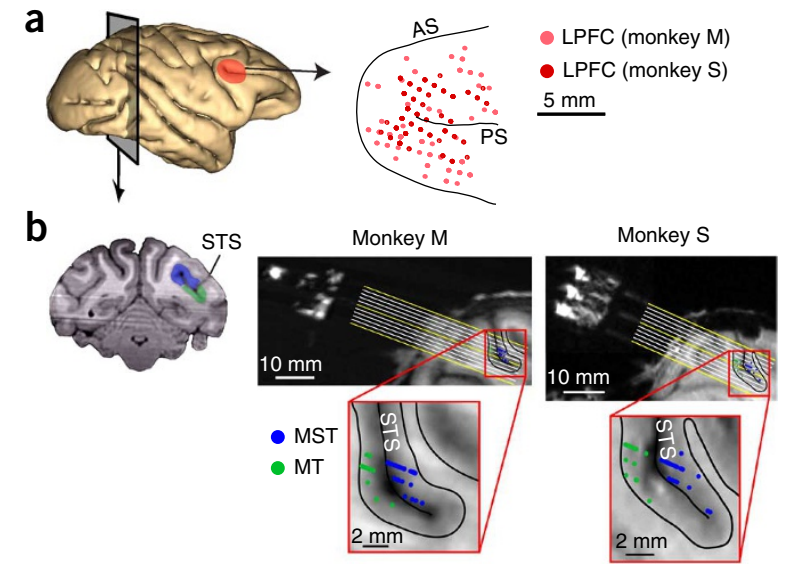

(Fig. 2e,g), which suggests that they better represent a memorized direction than the direction of a present stimulus.

We found direction selectivity in a total of 109 neurons in MT (97\%), 218 in MST (88\%) and 86 in LPFC (32\%). From these neurons, $100 \%$ in MT, $93 \%$ in MST and 70\% in LPFC showed sensory selectivity, while $8 \%$ in MT, 36\% in MST and 55\% in LPFC showed delay selectivity (Fig. 3g). Both monkeys showed similar results (Supplementary Fig. 2a,b). All percentages, except the percentage of delay-selective MT neurons, were significantly higher than expected by chance (permutation tests, $P<0.05$ ). A one-factor ANOVA comparing mean firing rates over each entire task period between sample directions yielded similar results (Supplementary Fig. 2c). All analyses hereafter were performed on sensory-selective and/or delay-selective neurons.

To measure how well neurons discriminated between motion directions (discriminability), we computed the area under the receiver operating characteristics curve (auROC) between activity in trials with the sample moving in the neuron's preferred and leastpreferred directions (Fig. $\mathbf{2 b}-\mathbf{g}$ and Online Methods) ${ }^{21}$. Upon sample onset, discriminability increased in all three areas but did so more rapidly and reached higher values in MT, followed by MST and then by LPFC (Fig. 3b,d,f). During the sample period, the discriminability strength (i.e., mean auROC; Fig. 3h) and duration (i.e., time that the auROC remained above chance value; Fig. 3a-c,i and Supplementary Fig. 3a,b), were higher in MT than MST (strength: unpaired $t$-test, $t=6.16, P<<0.001$; duration: unpaired $t$-test, $t=3.34, P<<0.001, t$-tests), and higher in MST than LPFC (strength: unpaired $t$-test,

Figure 2 Firing rate across task periods for example neurons in MT, MST and LPFC. (a) Visual display during all task periods. (b-g) Mean firing rate ( \pm s.e.m.; $n \geq 50$ ) over time in trials with each of the four sample directions (color-coded arrows) for neuron examples in MT (b,c), MST (d,e) and LPFC $(\mathbf{f}, \mathbf{g})$. Each neuron's preferred direction is shown in red. Gray area shows corresponding auROC over time (right axis label). In c, the test stimuli, but not the sample, were placed inside the neuron's receptive field, and colors during the test period represent test directions. 
Figure 3 Direction discriminability in MT, MST and LPFC. $(\mathbf{a}, \mathbf{c}, \mathbf{e})$ Time periods with significant direction discriminability (auROC) above (blue) and below (red) 0.5 for all neurons in MT (a), MST (c) and LPFC (e). (b,d,f) Average auROC across MT (b), MST (d) and LPFC ( $f$ ) neurons over time as a function of the percentage of averaged neurons (organized from maximum to minimum auROC in each time bin). Sample and delay periods used for analysis are indicated. (g) Percentage of selective neurons with sensory selectivity (upright bars) and delay selectivity (inverted bars). Because these percentages are computed from selective neurons, the bar overlap represents neurons with both sensory and delay selectivity. $(\mathbf{h}, \mathbf{i})$ Mean auROC (h) and percentage bins with significant auROC (i) during the sample and delay among all (light bars) and the top $10 \%$ (dark bars) of the neurons selective during each corresponding period. In $\mathbf{g}-\mathbf{i}$, dashed white lines show the values expected by chance. Error bars, s.e.m.

$t=5.99, P<<0.001$; duration: unpaired $t$-test, $t=5.55, P<0.01, t$-tests $)$. This indicates that the representation of motion direction of a present stimulus is strongest in MT, weaker downstream in MST and weakest in LPFC.

Immediately after the sample offset, discriminability in MT neurons quickly dropped and remained at chance values throughout the delay (Fig. 3a,b). In contrast, in many MST and LPFC neurons, it remained high (Fig. 3c-f). Delay-period discriminability in MST was as strong as

(Fig. 3h; unpaired $t$-test, $t=-1.51, P=0.13$ ) and lasted significantly longer than (Fig. 3i and Supplementary Fig. 3c,d; unpaired $t$-test, $t=2.20, P=0.03, t$-test) in LPFC, persisting throughout the entire delay in $22 \%$ of MST and $11 \%$ of LPFC delay-selective neurons. There was a small but significant decrease in discriminability by the end of the longest delay ( $2 \mathrm{~s})$ in LPFC $(0.036$ decrease, $P=0.04$, paired sample $t$-test, $t=1.90$ ) but not in MST (0.014 decrease, $P=0.08$, paired-sample $t$-test, $t=1.42$ ). These results indicate that sustained activity in MST encoded the memorized direction more robustly over time than in LPFC.

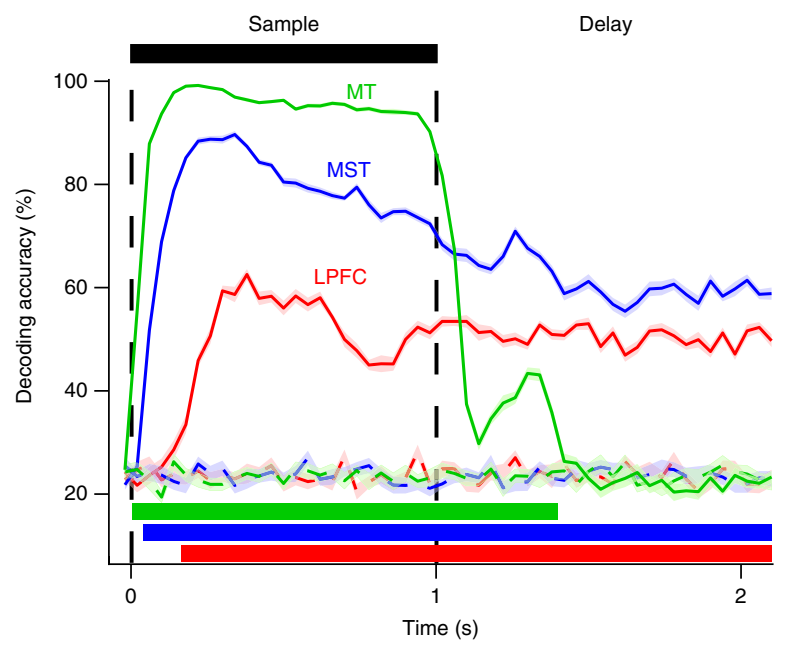

In contrast, in the few MT neurons classified as delay-selective, discriminability was only weak and brief (Fig. 3h,i and Supplementary Fig. 3c,d), and was not significantly different in strength (unpaired $t$-test, $t=0.76, P=0.45$ ) or duration (unpaired $t$-test, $t=1.04, P=0.30$ ) from that of neurons in which the test stimuli, rather than the sample, were placed inside the receptive field (Fig. 2c). Likewise, of the $12 \mathrm{MT}$ neurons recorded with the sample and test at the same location inside the receptive field, none were delay-selective, and their mean discriminability was 0.54 , as for all other MT neurons. In contrast, of the 30 MST neurons recorded with the sample and test inside the receptive field, $50 \%$ were delay-selective; their mean discriminability was 0.64 , the same value as for all other delay-selective MST neurons. Thus, sustained activity encoding the memorized motion directions was present in MST and LPFC but not in MT.

To estimate how strongly the activity of neuronal populations in each area encoded the sample direction, we performed linear discriminant analysis (Online Methods). In contrast to ROC analysis, which measures the ability of each neuron to discriminate between two of the four sample directions (preferred and least-preferred), linear discriminant analysis estimates the ability of an entire population of recorded neurons to discriminate among the four directions (decoding accuracy).

Figure 4 Direction decoding accuracy for the populations of MT, MST and LPFC neurons. Mean ( \pm s.e.m.; $n=50$ ) sample direction decoding accuracy (percentage of correctly decoded trials) over time for the neuronal populations in MT (green), MST (blue) and LPFC (red). Dashed lines, decoding accuracy expected by chance (Online Methods). Horizontal color bars show periods with significant decoding accuracy for each area. 
Figure 5 Relationship between task performance and delay-period direction discriminability of MST and LPFC neurons. (a,b) Mean firing rate of example MST (a) and LPFC (b) neurons over time in correct and error trials with preferred and least-preferred sample direction. Colored areas show difference in activity between preferred and least-preferred sample trials. (c,d) Delay-period auROC of delay-selective neurons in MST (c) and LPFC (d) in correct versus error trials. Gray, identity line. (e) Mean difference ( \pm s.e.m.; MST, $n=66$ and LPFC, $n=40$ ) in delayperiod auROC between correct and error trials $\left(\triangle \mathrm{auROC}=\mathrm{auROC}_{\text {correct }}\right.$ -

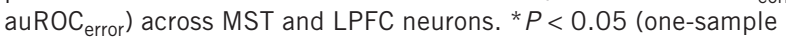
$t$-tests; unpaired $t$-test between areas).

In both MST and LPFC, population decoding accuracy increased after sample onset, and remained well above chance and relatively stable throughout the entire delay period (Fig. 4). Decoding accuracy was significantly higher in MST than LPFC not only during the sample presentation but also throughout all of the delay (Bonferronicorrected $t$-test), which indicates that the memorized directions were more strongly represented by the population of neurons in MST than in LPFC. In MT, decoding accuracy during the sample period was higher than in MST and LPFC. After the sample offset, however, it quickly dropped and remained at chance values for most of the delay period. Therefore, the memorized directions were robustly encoded in the population activity in MST and LPFC but not in MT.

Decoding accuracy in MT showed a brief and weak rebound after the sample offset, which coincided with the occurrence of a transient selectivity inversion in some neurons (lower responses to the preferred direction than to the least-preferred direction; Fig. 3a). This effect has been reported after visual stimulation in tasks with no memory requirements or during anesthesia and is likely a consequence of rapid neural adaptation ${ }^{22,23}$. Periods of inverted selectivity were also present in some MST neurons, but we observed them throughout the entire delay (Fig. 3c). In LPFC, we rarely observed this phenomenon (Fig. 3e).

\section{Sustained activity is linked to task performance}

A hypothesis derived from our results is that the direction-selective sustained activity of MST and LPFC neurons played a role in the animals' performance of the working memory task. This hypothesis predicts that delay-period discriminability should be reduced in error
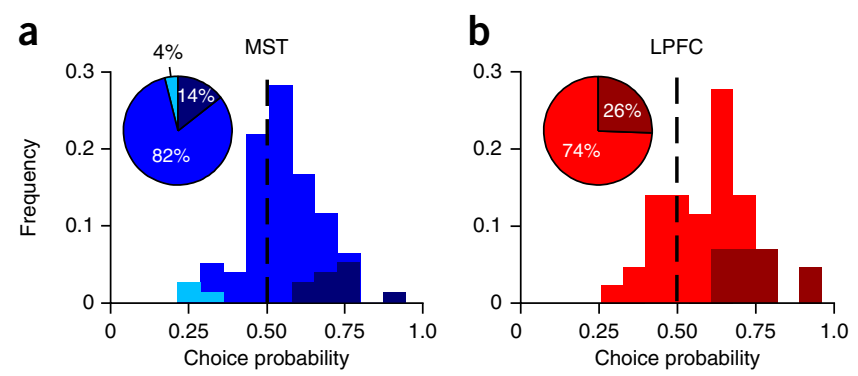

C

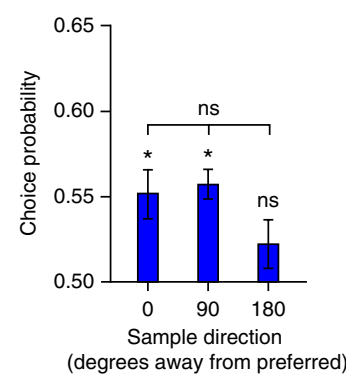

d

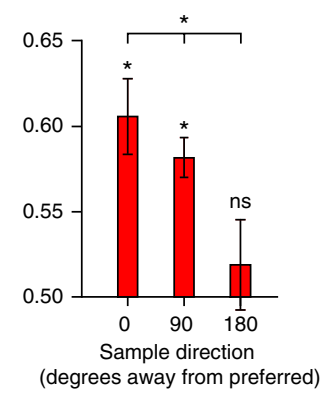

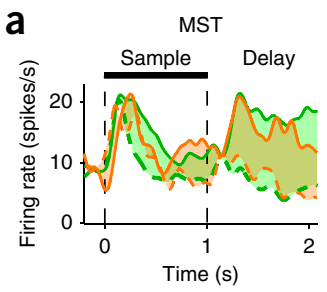
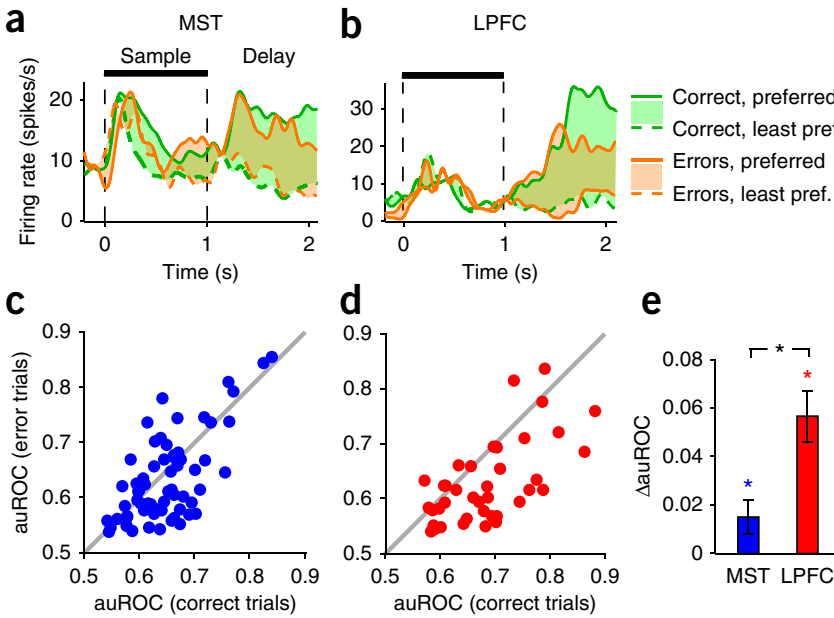

e

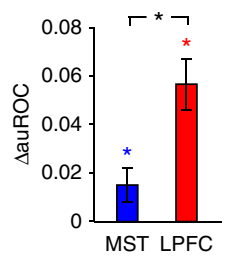

relative to correct trials and that trial-to-trial variations in sustained activity should correlate with the animals' performance.

For the example MST and LPFC neurons in Figure 5a,b, delay activity in trials with the sample in the neurons' preferred direction was higher in correct trials than in error trials. As a consequence, the difference in activity between preferred and least-preferred sample trials was larger in correct trials than in error trials. Across neurons and in both areas, the difference in auROC between correct and error trials was significantly higher than 0 , which indicated that discriminability during the delay period was reduced in error trials relative to correct trials (one-sample $t$-tests; MST: $t=2.12, P=0.02$ and LPFC: $t=5.37, P<0.001)$. This reduction was significantly larger in LPFC than MST (Fig. 5c-e; unpaired $t$-test, $t=1.79, P=0.038$ ). This suggests that the animals' performance was linked to how strongly MST and LPFC neurons encoded the memorized direction, and that this link was stronger in LPFC than in MST.

We then examined whether trial-to-trial variations in sustained activity correlated with the animals' performance and whether this relationship depended on each neuron's direction preference with respect to the memorized sample direction. We compared the distributions of firing rates during the delay period between correct and error trials using choice-probability analysis ${ }^{24}$. We did this independently for trials with the sample moving in the neurons' preferred, least-preferred and intermediate directions (Online Methods). Choice probability was significantly higher than expected by chance in $25 \%$ of LPFC neurons and 14\% of MST neurons, and significantly lower in $0 \%$ of LPFC neurons and $4 \%$ of MST neurons (Fig. $\mathbf{6 a}$,b; permutation test, $P<0.05)$. Across all neurons, mean choice probability during the delay period in preferred-sample trials was significantly higher than chance in both areas (one-sample $t$-tests; MST: $t=3.53, P<0.001$ and LPFC: $t=4.76, P<0.001)$ and was significantly higher in LPFC

Figure 6 Choice probability of delay activity in MST and LPFC neurons. $(\mathbf{a}, \mathbf{b})$ Frequency histogram of delay period choice probability among delay-selective MST (a) and LPFC (b) neurons in preferred-sample trials. Vertical black dashed lines show chance choice probability. Color tones represent neurons with choice probability significantly above (darker tones), significantly below (lighter tones) and not significantly different from (middle tones) that expected by chance. (c,d) Mean choice probability ( \pm s.e.m.; MST, $n=74$ and LPFC, $n=43$ ) among delay-selective MST (c) and LPFC (d) neurons as a function of sample direction with respect to each neuron's preferred direction. ${ }^{*} P<0.05 ;$ ns, nonsignificant (one-sample $t$-tests; repeated-measures ANOVA across directions). 
Figure 7 Direction discriminability of LFP power in MT during working memory. (a) Mean normalized LFP spectrogram of an example recording site in MT during trials with preferred (top) and least-preferred (bottom) sample directions. Black horizontal bars and dashed lines delineate sample period. Frequency band ranges are color-coded: theta $(\theta)=4-8 \mathrm{~Hz}$, alpha $(\alpha)=8-12 \mathrm{~Hz}$, beta $(\beta)=12-25 \mathrm{~Hz}$, low gamma $\left(\gamma_{\mathrm{L}}\right)=25-55 \mathrm{~Hz}$ and high gamma $\left(\gamma_{H}\right)=65-135 \mathrm{~Hz}$. (b,c) For each frequency band, percent LFP sites in MT for which the LFP power auROC in the delay period (b), and mean LFP auROC ( \pm s.e.m.; $\theta, n=8$; $\alpha, n=11 ; \beta, n=9 ; \gamma_{\mathrm{L}}, n=9 ; \gamma_{\mathrm{H}}, n=12$ ) among selective sites (c).

than in MST (Fig. 6a-d; unpaired $t$-test, $t=2.10, P=0.02$ ). In both areas, mean choice probability across significant neurons remained significantly above chance throughout most of the delay period (Supplementary Fig. 4). These results show that variations in the neurons' sustained activity correlated with the animals' performance in both areas, but more so in LPFC than MST. Finally, in LPFC but not MST, mean choice probability decreased significantly as a function of the difference between the neurons' preferred direction and the memorized direction (Fig. 6c,d; repeated-measures ANOVA; LPFC: $F=4.78, P=0.01$ and MST: $F=2.41, P=0.09$ ). This suggests that the link of each LPFC neuron to behavior depends on the similarity between its preferred feature and the memorized feature.

\section{LFP power in MT encodes memorized directions}

Given the existence of feedback projections from MST and LPFC to $\mathrm{MT}^{18,25}$, it may appear surprising that the sustained activity of MST and LPFC neurons did not cause firing-rate increases in MT neurons during the delay period. One possible explanation is that during this period, feedback signals from MST and/or LPFC modulate synaptic activity in MT, sufficiently to cause changes in local field potentials (LFPs) that reflect the memorized directions but not strongly enough to cause increases in neuronal firing.
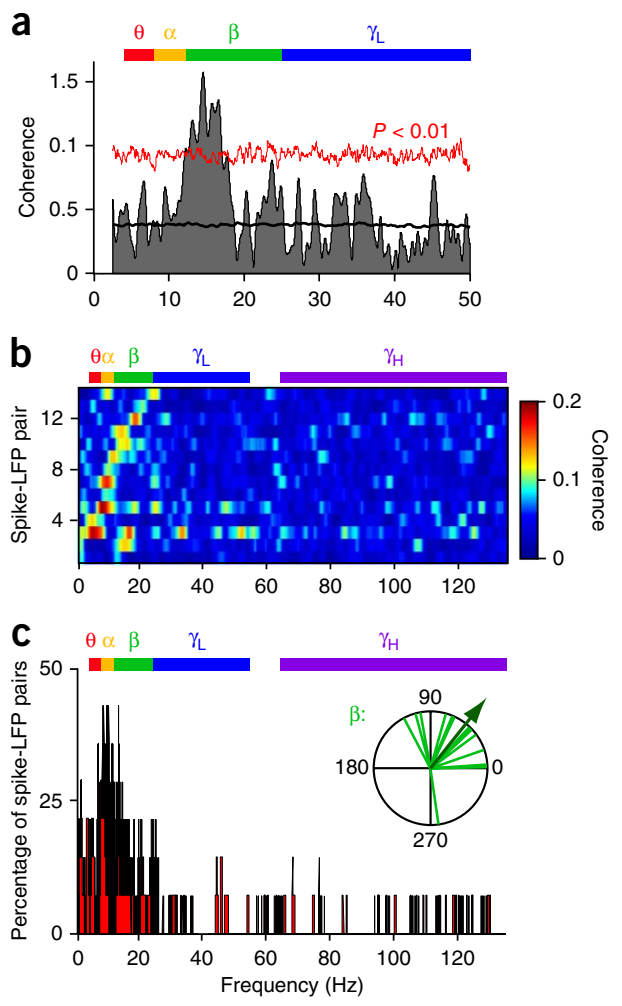
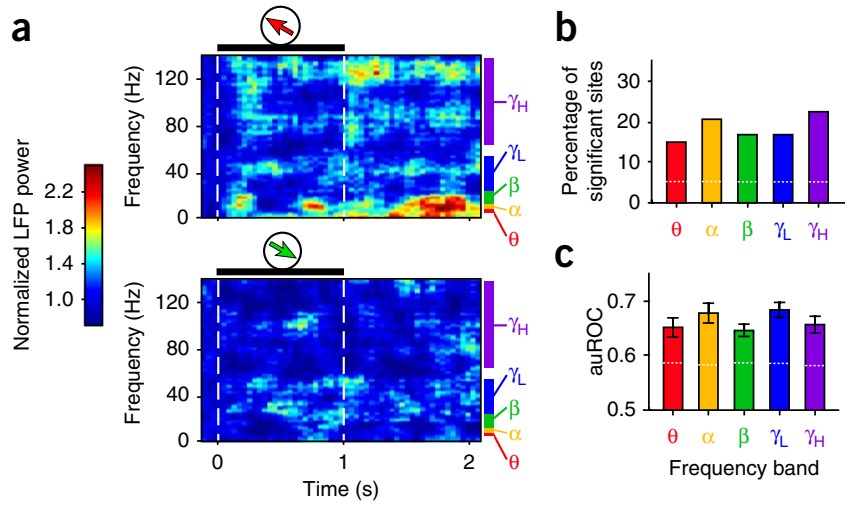

Indeed, in many MT recording sites, such as the example in Figure 7a, power in several LFP frequencies differed in response to different memorized sample directions. This effect was not due to residual activity (spikes or LFPs) caused by the sample, as we observed it not only in sites recorded with the sample inside the receptive field but also in sites recorded with the sample outside, such as that shown in Figure 7a. For each frequency band ${ }^{26}$ (Online Methods), we measured the delay-period direction discriminability of the LFP power in each MT site (i.e., the auROC between power in preferred and leastpreferred sample trials). In all bands, the percentage of sites for which the auROC was significantly higher than expected by chance ranged between $14 \%$ and $22 \%$ (Fig. 7 b; permutation test, $P<0.05$ ). These values were significantly higher than the percentage of false positives (population permutation test, $P<0.05$ ). The mean auROC across significant sites in all frequency bands ranged between 0.64 and 0.67 , and were all significantly above the values expected by chance (permutation test, $P<0.05$, Fig. $7 \mathrm{c}$ ). These results indicate that in MT, the amplitude of LFP oscillations reflects the memorized directions, despite the absence of direction-selective sustained spiking activity.

We conducted the same analysis in MST and LPFC. In both areas, the percentage of sites with delay-period direction discriminability in the LFP power was higher than the percentage of false positives for all frequency bands. These values, as well as the mean auROC across significant sites, were similar to those in MT (Supplementary Fig. 5). Thus, in MST and LPFC, both sustained spiking activity and LFP power encoded the memorized motion directions.

Spike-field synchrony between LPFC and MT during the delay To examine whether the LFP activity observed in MT during the delay period was driven by feedback signals from LPFC, we measured phase coherence between simultaneously recorded spikes from LPFC neurons and LFPs from MT recording sites. For the example pair (LPFC neuron and MT site) shown in Figure 8a, coherence was above that expected by chance in the low frequencies (randomized surrogates test, $P<0.01)$, particularly in the beta band $(12-25 \mathrm{~Hz})$. We observed significant coherence in $12.5 \%$ (14 of 112) of the recorded LPFC-MT

Figure 8 Spike-field synchrony between LPFC and MT during working memory. (a) Spike-field phase coherence (shaded area) of example LPFC-MT pair as a function of LFP frequency; mean coherence among randomized surrogates (black line) and confidence limit of $P<0.01$ (red line; randomized surrogates test). (b) Spike-field coherence as a function of LFP frequency for all significantly coherent pairs, sorted by frequency at peak coherence. (c) Among coherent pairs, percent reaching significant coherence at each frequency. Inset, phase of coherence for all coherent pairs in the beta band (in degrees). Arrow indicates mean phase across pairs. Frequency bands are color-coded. 
pairs. For all of these pairs, coherence was high and reached significance in the low-frequency bands (theta, alpha and beta; Fig. $\mathbf{8 b}, \mathbf{c}$ ), peaking in the range of 7-15 Hz. We repeated the same analysis on pairs showing significant coherence after shuffling the trial order, which destroyed the trial-to-trial simultaneity of LFP and spike signals. The coherence peak in the lower bands was completely absent (Supplementary Fig. 6), which indicates that such peak was due to the real-time interaction of LFP and spike signals, and was not an artifact of any other property of these signals.

In the theta, alpha and beta bands, the phases of coherence among significantly coherent pairs were non-uniformly distributed along the LFP oscillatory cycle (Rayleigh test, $P<0.05$ ), showing the maximal concentration in the beta band (concentration parameter $\kappa=1.64$ ), mostly in the rising phase of the cycle (Fig. 8c; mean phase $=51^{\circ}$, significantly different from $0^{\circ}$; one-sample test for circular data, $P=0.005)$. Thus, the phase relationship between LPFC spikes and LFP oscillations in MT was consistent across pairs.

Lastly, we examined whether there was a relationship between LPFCMT synchrony and the animals' task performance. Among synchronous pairs, the percentage with significant coherence was decreased in error relative to correct trials by $54 \%$ in the theta band, $64 \%$ in the alpha band and $65 \%$ in the beta band (Fig. 8c). Our results show that during the delay period, the spiking activity of a proportion of LPFC neurons was synchronized with low-frequency LFP oscillations in MT and that lower task performance was associated with reduced synchrony. Coupling of low-frequency oscillatory activity during similar tasks has been previously observed between prefrontal and ventral visual cortex ${ }^{27}$, and between prefrontal and parietal areas ${ }^{28}$.

\section{DISCUSSION}

\section{Emergence of feature-selective sustained activity}

Our study demonstrated that while monkeys memorized the motion direction of a previous stimulus, sustained spiking activity encoding the memorized direction was absent in early visual area MT. Together with previous studies reporting weak, transient or no coding of memorized features in early visual neurons during similar tasks ${ }^{9-12}$, our results argue against the hypothesis that neurons in early visual cortex are involved in the coding of working memory representations of single visual features. Our observation that MT neurons robustly encoded the sample direction exclusively during the sample period suggests that spiking activity in early visual areas mainly encodes the current visual input.

Given the direct feed-forward and feedback connectivity between MT and $\mathrm{MST}^{18}$, it was surprising that feature-selective sustained activity was robustly present in MST but absent in MT. Our results indicate that along the dorsal visual pathway, this sustained activity sharply emerges as a de novo property of MST neurons. Moreover, we found that some MST neurons had sensory selectivity but not delay selectivity, others had delay selectivity but not sensory selectivity and yet others had both (Fig. 3g). This indicates that information about present and past stimuli remains segregated in some neurons and coexists in others, and suggests that along the dorsal visual pathway, the transformation of sensory representations of motion direction into mnemonic representations occurs in the MST circuitry. Such transformation may also involve LPFC, as neurons in this area also showed heterogeneity of coding.

The observation that the memorized motion direction was encoded by neurons in LPFC is further evidence that, in addition to locations ${ }^{2}$ and complex objects ${ }^{3}$, these neurons also encode mnemonic representations of single visual features. Working memory coding of visual-motion speed gradients ${ }^{29}$ and vibrotactile stimuli ${ }^{30}$ has also been reported. On the other hand, our observation seems at odds with results from human functional imaging experiments showing the inability to decode the contents of working memory using multivoxel pattern classification analysis of BOLD signals recorded from $\mathrm{LPFC}^{15}$. This may be explained by considering that LPFC lacks a topographical organization of feature coding at a scale that can be detected with the coarse resolution of functional imaging.

Consistent with our results, a previous study ${ }^{10}$ found no sustained activity in MT neurons during a delayed match-to-sample task for motion direction, and observed only brief and transient activity mostly restricted to the first few hundred milliseconds after sample offset. This is likely due to residuals of the response to the sample, given the observations that sensory responses are followed by a brief period of decay toward baseline $\mathrm{e}^{31,32}$ and by rapid neural adaptation ${ }^{9,22,23}$, even during tasks that do not require memory or during anesthe$\operatorname{sia}^{22,23,31,32}$.

The same study ${ }^{10}$ reported that no LPFC neurons showed sustained activity persistently encoding the memorized directions. They proposed that in LPFC, as well as in MT, brief and transient instances of weak coding in individual neurons during the delay period could be integrated, yielding a population code that reliably represents the memorized direction over time. However, such mechanism might not be necessary, given our finding that in both MST and LPFC, a substantial number of neurons robustly encoded the memorized directions throughout the entire delay. Moreover, population decoding accuracy in MT dropped to chance values during the delay period, which suggests that a representation of the memorized directions is absent even when integrating the activity of multiple MT neurons (Fig. 4).

Another study reported that the firing rate of neurons in early visual area V1 was modulated by the memorized location of a motion-defined figure previously presented on a moving background texture $^{13}$. This effect, however, is different from the sustained activity reported here in the absence of visual stimulation, as it required the background texture to constantly stimulate the neuron's receptive field during the delay period.

One issue that has been a matter of debate is whether the sustained activity of LPFC neurons reflects the allocation of attention rather than the maintenance of memories ${ }^{33}$. The sustained activity we found in MST and LPFC may reflect either the maintenance of mnemonic representations of motion direction or the allocation of attention to (or monitoring of) such representations ${ }^{20}$. These two different functions have been considered as components of the broader construct of working memory ${ }^{1}$. Our behavioral paradigm was not designed to discriminate between them. Further electrophysiological studies using retro-cueing ${ }^{34}$ tasks are needed to clarify this issue.

\section{Role of MST and LPFC sustained activity in working memory}

A puzzling finding in our study was that neurons in both MST and LPFC redundantly encoded memorized motion directions. Redundancy has also been observed between parietal and prefrontal cortices during memory for visuospatial locations ${ }^{35}$ and visual feature categories ${ }^{36}$, and between inferotemporal and prefrontal cortices during memory for complex objects ${ }^{3}$. One likely explanation for this redundancy is that the maintenance of working memory representations results from the coordinated activity of an interconnected network of brain areas, each of them playing a different and complementary role. For example, evidence of different roles of the lateral intraparietal area and LPFC in the filtering of distracting information during working memory tasks has been recently reported ${ }^{37}$. Delay activity in LPFC neurons is more robust to distractors than in 
lateral intraparietal neurons. We did not test the effect of distracting information during the delay period on MST and LPFC neurons, but it is likely that activity in MST is more sensitive to distractor interference than in LPFC.

One possible scenario is that MST maintains a robust representation of the sample direction, which can be 'read out' by LPFC and integrated with signals encoding reward value and the allocation of attention in order to produce a meaningful behavioral response. Consistent with this hypothesis, LPFC neurons encode the behavioral choice in a delayed match-to-sample task ${ }^{29}$. In our study, representations of the memorized direction in MST neurons lasted longer than, and at the population level were stronger than, in LPFC, challenging the view of LPFC as the major contributor to the maintenance of working memory ${ }^{3}$. However, variations in sustained activity were more predictive of task performance in LPFC than MST, consistent with the notion that activity becomes more closely linked to behavior further downstream along the chain of visuomotor processing ${ }^{30}$. However, it may also reflect the influence of attention and other variables in choice-probability measurements, which may be heterogeneous across different brain areas ${ }^{38}$.

\section{Modulation of LFPs in area MT}

Despite the absence of sustained spiking activity in MT, we found that the memorized direction was encoded in the amplitude of LFP oscillations. Given that LFPs represent the overall synaptic activity around the recorded site ${ }^{39}$ (owing to feed-forward, feedback and local neuronal interactions), and given the absence of feed-forward visual inputs into MT neurons during the delay period of our task, our results suggest that this effect may be due to feedback signals from MST or LPFC ${ }^{25}$, sufficient to modulate MT synaptic activity but insufficient to increase firing rates. Dissociations between LFP and spiking activity are now established ${ }^{12,40}$. If functionally relevant, the information reflected in the LFPs of MT must exit the area and hence must be observable in spiking activity. As it is not observed at the single-unit level, it might be manifested in spike synchrony ${ }^{41}$.

Supporting this hypothesis, during the delay period, spikes of LPFC neurons were phase-coherent with low-frequency LFP oscillations in MT. Moreover, in agreement with a previous report ${ }^{27}$, when LPFC-MT synchrony was reduced, the monkeys were more likely to subsequently produce an incorrect response. This synchrony may represent a top-down mechanism by which sustained activity in higher-order areas selectively modulates the responses of early visual neurons to incoming sensory inputs, biasing visual perception ${ }^{42-45}$. Supporting this hypothesis, during a delayed match-to-sample task for motion direction, MT neuron responses to the test stimulus are influenced by the direction of the remembered sample ${ }^{42}$. Whether the same phenomenon is present in our data will require an analysis of the test responses as a function of the sample direction.

Coding of the memorized direction in the LFPs from MT may explain why the contents of visual working memory can be decoded from BOLD signals recorded in human early visual cortex using multivoxel pattern classification analysis ${ }^{8,14,15}$. Given that the amplitude of BOLD signals correlates with LFP amplitude in the absence of changes in neuronal firing rates ${ }^{40}$, these functional magnetic resonance imaging studies have likely measured a BOLD correlate of the LFP modulation we observed in early visual cortex ${ }^{8,46}$.

Implications for mechanisms and models of working memory Current models of working memory networks propose that recurrent excitatory connections in high-order association areas such as LPFC underlie sustained spiking activity ${ }^{47-49}$. Our results suggest that such mechanisms may operate as early as in MST. In contrast, in early visual areas such as MT, strong feedforward inputs from upstream areas, relatively weak feedback inputs from downstream areas, and stronger lateral inhibitory interactions between neurons ${ }^{50}$, may cause spiking activity to increase vigorously in response to retinal inputs but rapidly decay in their absence. Alternatively, it may be that the cortical architecture characteristic of LPFC is not present in MST, and sustained activity in MST arises via LPFC feedback. However, visual responses to the sample arise in MST earlier than in LPFC and persist throughout the delay period, which suggests that sustained activity may be intrinsically generated in MST rather than inherited from LPFC and may instead be transferred from MST to LPFC through feedforward inputs.

Our results indicate that the properties of the cortical architecture in MST that allow neurons to generate sustained activity during working memory are absent immediately upstream, in MT. This suggests a sharp transition in cortical architecture between MT and MST, which may also be present at similar processing stages along other sensory processing streams $s^{5,30}$. We propose that the boundary between the two architectures may be important for the brain to distinguish representations of current sensory experiences from those imagined or memorized, a function that is impaired in schizophrenia and other hallucinatory mental disorders.

\section{METHODS}

Methods and any associated references are available in the online version of the paper.

Note: Any Supplementary Information and Source Data files are available in the online version of the paper.

\section{ACKNOWLEDGMENTS}

This study was supported by grants awarded to J.C.M.-T. from the Canadian Institutes of Health Research (CIHR), the Canada Research Chairs Program (CRC) and the EJLB Foundation. We thank M. Schneiderman for assistance with electrophysiological recordings, and W. Kucharski and S. Nuara for technical assistance.

\section{AUTHOR CONTRIBUTIONS}

D.M.-H. and J.C.M.-T. designed the experiments. D.M.-H. and S.T. conducted the experiments. D.M.-H. analyzed the data. D.M.-H. and J.C.M.-T. wrote the article. All authors discussed the results and commented on the manuscript.

\section{COMPETING FINANCIAL INTERESTS}

The authors declare no competing financial interests.

Reprints and permissions information is available online at http://www.nature.com/ reprints/index.html.

1. Baddeley, A. Working memory: theories, models, and controversies. Annu. Rev. Psychol. 63, 1-29 (2012).

2. Funahashi, S., Bruce, C.J. \& Goldman-Rakic, P.S. Mnemonic coding of visual space in the monkey's dorsolateral prefrontal cortex. J. Neurophysiol. 61, 331-349 (1989).

3. Miller, E., Erickson, C. \& Desimone, R. Neural mechanisms of visual working memory in prefrontal cortex of the macaque. J. Neurosci. 16, 5154-5167 (1996).

4. Andersen, R.A., Essick, G.K. \& Siegel, R.M. Neurons of area 7 activated by both visual stimuli and oculomotor behavior. Exp. Brain Res. 67, 316-322 (1987).

5. Miller, E.K., Li, L. \& Desimone, R. A neural mechanism for working and recognition memory in inferior temporal cortex. Science 254, 1377-1379 (1991).

6. Constantinidis, C. \& Procyk, E. The primate working memory networks. Cogn. Affect. Behav. Neurosci. 4, 444-465 (2004).

7. Pasternak, T. \& Greenlee, M.W. Working memory in primate sensory systems. Nat. Rev. Neurosci. 6, 97-107 (2005).

8. Harrison, S.A. \& Tong, F. Decoding reveals the contents of visual working memory in early visual areas. Nature 458, 632-635 (2009).

9. Bisley, J.W., Zaksas, D., Droll, J.A. \& Pasternak, T. Activity of neurons in cortical area MT during a memory for motion task. J. Neurophysiol. 91, 286-300 (2004).

10. Zaksas, D. \& Pasternak, T. Directional signals in the prefrontal cortex and in area MT during a working memory for visual motion task. J. Neurosci. 26, 11726-11742 (2006). 
11. Ferrera, V.P., Rudolph, K.K. \& Maunsell, J.H. Responses of neurons in the parietal and temporal visual pathways during a motion task. J. Neurosci. 14, 6171-6186 (1994).

12. Lee, H., Simpson, G.V., Logothetis, N.K. \& Rainer, G. Phase locking of single neuron activity to theta oscillations during working memory in monkey extrastriate visual cortex. Neuron 45, 147-156 (2005).

13. Super, H., Spekreijse, H. \& Lamme, V.A. A neural correlate of working memory in the monkey primary visual cortex. Science 293, 120-124 (2001).

14. Sneve, M.H., Alnaes, D., Endestad, T., Greenlee, M.W. \& Magnussen, S. Visual short-term memory: activity supporting encoding and maintenance in retinotopic visual cortex. Neuroimage 63, 166-178 (2012).

15. Riggall, A.C. \& Postle, B.R. The relationship between working memory storage and elevated activity as measured with functional magnetic resonance imaging. J. Neurosci. 32, 12990-12998 (2012).

16. Born, R.T. \& Bradley, D.C. Structure and function of visual area MT. Annu. Rev. Neurosci. 28, 157-189 (2005).

17. Saito, H. et al. Integration of direction signals of image motion in the superior temporal sulcus of the macaque monkey. J. Neurosci. 6, 145-157 (1986).

18. Boussaoud, D., Ungerleider, L.G. \& Desimone, R. Pathways for motion analysis: cortical connections of the medial superior temporal and fundus of the superior temporal visual areas in the macaque. J. Comp. Neurol. 296, 462-495 (1990).

19. Angelaki, D.E., Gu, Y. \& Deangelis, G.C. Visual and vestibular cue integration for heading perception in extrastriate visual cortex. J. Physiol. (Lond.) 589, 825-833 (2011).

20. Petrides, M. Lateral prefrontal cortex: architectonic and functional organization. Phil. Trans. R. Soc. Lond. B 360, 781-795 (2005).

21. Lennert, T. \& Martinez-Trujillo, J. Strength of response suppression to distracter stimuli determines attentional-filtering performance in primate prefrontal neurons. Neuron 70, 141-152 (2011).

22. Van Wezel, R.J. \& Britten, K.H. Motion adaptation in area MT. J. Neurophysiol. 88, 3469-3476 (2002).

23. Glasser, D.M., Tsui, J.M., Pack, C.C. \& Tadin, D. Perceptual and neural consequences of rapid motion adaptation. Proc. Natl. Acad. Sci. USA 108, E1080-E1088 (2011).

24. Britten, K.H., Newsome, W.T., Shadlen, M.N., Celebrini, S. \& Movshon, J.A. A relationship between behavioral choice and the visual responses of neurons in macaque MT. Vis. Neurosci. 13, 87-100 (1996).

25. Ninomiya, T., Sawamura, H., Inoue, K. \& Takada, M. Segregated pathways carrying frontally derived top-down signals to visual areas MT and V4 in macaques. J. Neurosci. 32, 6851-6858 (2012).

26. Khawaja, F.A., Tsui, J.M. \& Pack, C.C. Pattern motion selectivity of spiking outputs and local field potentials in macaque visual cortex. J. Neurosci. 29, 13702-13709 (2009).

27. Liebe, S., Hoerzer, G.M., Logothetis, N.K. \& Rainer, G. Theta coupling between V4 and prefrontal cortex predicts visual short-term memory performance. Nat. Neurosci. 15, 456-462 (2012).

28. Salazar, R.F., Dotson, N.M., Bressler, S.L. \& Gray, C.M. Content-specific frontoparietal synchronization during visual working memory. Science 338, 1097-1100 (2012).

29. Hussar, C.R. \& Pasternak, T. Common rules guide comparisons of speed and direction of motion in the dorsolateral prefrontal cortex. J. Neurosci. 33, 972-986 (2013).

30. Hernandez, A. et al. Decoding a perceptual decision process across cortex. Neuron 66, 300-314 (2010).
31. Priebe, N.J., Churchland, M.M. \& Lisberger, S.G. Constraints on the source of short-term motion adaptation in macaque area MT. I. the role of input and intrinsic mechanisms. J. Neurophysiol. 88, 354-369 (2002).

32. Osborne, L.C., Bialek, W. \& Lisberger, S.G. Time course of information about motion direction in visual area MT of macaque monkeys. J. Neurosci. 24, 3210-3222 (2004).

33. Lebedev, M.A., Messinger, A., Kralik, J.D. \& Wise, S.P. Representation of attended versus remembered locations in prefrontal cortex. PLoS Biol. 2, e365 (2004).

34. Nobre, A.C. et al. Orienting attention to locations in perceptual versus mental representations. J. Cogn. Neurosci. 16, 363-373 (2004).

35. Chafee, M.V. \& Goldman-Rakic, P.S. Matching patterns of activity in primate prefrontal area 8a and parietal area 7ip neurons during a spatial working memory task. J. Neurophysiol. 79, 2919-2940 (1998).

36. Swaminathan, S.K. \& Freedman, D.J. Preferential encoding of visual categories in parietal cortex compared with prefrontal cortex. Nat. Neurosci. 15, 315-320 (2012).

37. Suzuki, M. \& Gottlieb, J. Distinct neural mechanisms of distractor suppression in the frontal and parietal lobe. Nat. Neurosci. 16, 98-104 (2013).

38. Cohen, M.R. \& Newsome, W.T. Estimates of the contribution of single neurons to perception depend on timescale and noise correlation. J. Neurosci. 29, 6635-6648 (2009).

39. Mitzdorf, U. Current source-density method and application in cat cerebral cortex investigation of evoked potentials and EEG phenomena. Physiol. Rev. 65, 37-100 (1985).

40. Bartolo, M.J. et al. Stimulus-induced dissociation of neuronal firing rates and local field potential gamma power and its relationship to the resonance blood oxygen level-dependent signal in macaque primary visual cortex. Eur. J. Neurosci. 34 1857-1870 (2011).

41. Denker, M. et al. The local field potential reflects surplus spike synchrony. Cereb. Cortex 21, 2681-2695 (2011).

42. Lui, L.L. \& Pasternak, T. Representation of comparison signals in cortical area MT during a delayed direction discrimination task. J. Neurophysiol. 106, 1260-1273 (2011).

43. Mendoza, D., Schneiderman, M., Kaul, C. \& Martinez-Trujillo, J. Combined effects of feature-based working memory and feature-based attention on the perception of visual motion direction. J. Vision 11, 1-15 (2011).

44. Treue, S. \& Martinez Trujillo, J.C. Feature-based attention influences motion processing gain in macaque visual cortex. Nature 399, 575-579 (1999).

45. McAdams, C.J. \& Maunsell, J.H. Effects of attention on the reliability of individual neurons in monkey visual cortex. Neuron 23, 765-773 (1999).

46. Magri, C., Schridde, U., Murayama, Y., Panzeri, S. \& Logothetis, N.K. The amplitude and timing of the BOLD signal reflects the relationship between local field potential power at different frequencies. J. Neurosci. 32, 1395-1407 (2012).

47. Ardid, S., Wang, X.J. \& Compte, A. An integrated microcircuit model of attentional processing in the neocortex. J. Neurosci. 27, 8486-8495 (2007).

48. Goldman-Rakic, P.S. Cellular basis of working memory. Neuron 14, 477-485 (1995).

49. Lim, S. \& Goldman, M.S. Balanced cortical microcircuitry for maintaining information in working memory. Nat. Neurosci. 16, 1306-1314 (2013).

50. Haider, B., Hausser, M. \& Carandini, M. Inhibition dominates sensory responses in the awake cortex. Nature 493, 97-100 (2013). 


\section{ONLINE METHODS}

Animals. Two adult male rhesus monkeys (Macaca mulatta), 10 and 11 years old and weighing $8 \mathrm{~kg}$ and $9 \mathrm{~kg}$, participated in the experiments. The monkeys were rewarded with fruit juice for correctly performing each task trial $(300-600 \mathrm{ml}$ daily). At the end of each training and recording session, they also received fruits additionally to their daily food ration. We measured their body weight daily to ensure stable health conditions. All animal procedures complied with the Canadian Council of Animal Care guidelines and were approved by the McGill University Animal Care Committee.

Visual stimuli. Visual stimuli were generated using custom-made software on an Apple G4 computer and were back-projected onto a screen using a NEC WT610 video projector $(1,024 \times 760$ pixels resolution, $85 \mathrm{~Hz}$ refresh rate). The monkeys were positioned $57 \mathrm{~cm}$ away from the screen. Sample and test stimuli were composed of random dots moving linearly with $100 \%$ coherence within a virtual circular aperture $\left(13 \mathrm{~cd} / \mathrm{m}^{2}\right.$ dot luminance contrast, $0.17^{\circ}$ dot size, density of 4 dots/degree ${ }^{2}$ ). High motion coherence ensured that encoding of the sample direction in working memory was not ambiguous. The dots' speed was matched to the preferred speed of the recorded neuron, $2-32^{\circ} \mathrm{s}^{-1}$. The motion directions of the sample and tests were chosen from a set of four orthogonal directions and aligned so that one of them matched the neuron's preferred direction.

Behavioral task. We trained monkeys to perform a delayed match-to-sample task (Fig. 2a). During each trial, the monkey maintained gaze on a white fixation square (size $0.25^{\circ} \times 0.25^{\circ}$ ) at the center of the screen and pressed a button to initiate the trial. After $470 \mathrm{~ms}$, a sample stimulus with motion in one of four orthogonal directions was presented for $1,000 \mathrm{~ms}$. The sample was then removed and after a delay period of variable duration (from 1,200 ms to 2,000 ms), two test stimuli were sequentially presented for $590 \mathrm{~ms}$ each, with $590 \mathrm{~ms}$ in between. To receive a juice reward, the monkey was required to release the button during the presentation of a test with the same motion direction as the sample. This occurred in half of the trials for each test. If the monkey failed to do so, the trial was immediately terminated without a reward.

A set of four directions, from which the sample and test directions were chosen, was used for each recorded neuron (often differing from neuron to neuron within the same day). Thus, the monkeys could neither use long-term memory representations of these directions nor simply learn four fixed categories across sessions to solve the task. In trials in which the first test did not match the sample, the direction of this stimulus was randomly chosen from the other three directions in the set. A behaviorally irrelevant stimulus with $0 \%$ coherent motion and lower luminance contrast was presented simultaneously with the two tests on the opposite hemifield. The test and irrelevant stimulus locations were randomly swapped from trial to trial, preventing the monkeys from predicting the test location before its presentation. The irrelevant stimulus also served other experimental purposes unrelated to the results presented here. The variable delay prevented monkeys from anticipating the timing of the test onset.

Eye positions. We sampled eye position signals at a frequency of $200 \mathrm{~Hz}$ using a video-based eye tracker system (Eye Link 1000, SR Research). Monkeys were allowed to start a trial only when their gaze position fell within $1^{\circ}$ from the fixation point center. The trial was terminated without a reward if gaze position moved outside this area before the end of the trial.

Surgical preparation of the monkeys. Monkeys were implanted with titanium head posts that stabilized the head during recordings, and with two circular Cilux recording chambers $20 \mathrm{~mm}$ in diameter (Crist Instruments). One chamber was positioned on top of a circular craniotomy of the frontal bone that provided access to the right LPFC, specifically the region anterior to the arcuate sulcus, posterior and around the principal sulcus (centered at $30 \mathrm{~mm}$ anterior and $17 \mathrm{~mm}$ lateral in stereotactic coordinates). The other chamber was implanted on top of a craniotomy of the occipital bone, with its border $2 \mathrm{~mm}$ anterior to the occipital ridge and $2 \mathrm{~mm}$ lateral of the sagittal suture. The angle between the chamber's vertical axis and the horizontal plane was $20^{\circ}$. Electrode trajectories reached areas MT and MST in the right hemisphere according to MRI reconstructions (Fig. 1).

Anatomical localization of recording sites. An MRI scan was conducted on each monkey before the surgery in order to guide the positioning of the chamber.
After chamber implantation, a plastic grid (Crist Instruments) was positioned on top of each recording chamber and glass capillaries filled with mineral oil were positioned parallel to electrode trajectories at five different positions in the grid. An additional MRI scan was conducted to precisely locate the brain areas of interest with respect to the electrode trajectories. LPFC neurons were recorded by placing the electrode tip in positions around the principal sulcus, anterior to the arcuate sulcus. To record from MT, we positioned the electrode tip ventral and posterior to the superior temporal sulcus. To record from MST, the electrode tip was placed dorsal and anterior to the superior temporal sulcus. A reconstruction of the recording sites shows a clear segregation in the locations of the recorded MT and MST neurons, separated by the superior temporal sulcus (Fig. 1b). The recorded MST neurons were located within the dorsal region (MSTd).

Electrophysiological recordings. During each recording session, we made transdural penetrations with standard epoxy-insulated extracellular tungsten electrodes (FHC Inc; shank diameter $=500 \mu \mathrm{m}$ in LPFC and shank diameter $=125 \mu \mathrm{m}$ in MT and MST; impedance $=2-4 \mathrm{M} \Omega$ at $1 \mathrm{kHz}$ ). For LPFC recordings, a blunt guide tube positioned 5-10 $\mathrm{mm}$ from the recording electrode(s) served as the reference. For MT and MST recordings, a guide tube was lowered through the posterior craniotomy until it penetrated the dura, and the electrode was then lowered through the guide tube until it reached the desired depth. During each session, we recorded with one electrode placed in MT or MST and/or with one to four electrodes simultaneously in LPFC (separated by at least $2 \mathrm{~mm}$ ). A Plexon data acquisition system (MAP) was used to record, store and sort spike and LFP data (Plexon Inc.) $)^{21}$. LFP signals were band-pass-filtered between $0.7 \mathrm{~Hz}$ and $170 \mathrm{~Hz}$ and sampled at $1 \mathrm{kHz}$.

Characterizing spatial and motion tuning. The spatial and motion tuning properties of MT and MST neurons were characterized during trials in which the monkey responded to a contrast change in the fixation point while random dot stimuli with different locations, sizes, linear and spiral motion directions and speeds were presented in the visual periphery ${ }^{44}$. MT neurons were identified based on their linear motion direction selectivity, receptive fields size and laterality (contralateral to the recorded hemisphere). MST neurons were identified based on their linear and spiral motion direction selectivity, and receptive field size and position (considerably larger than for MT neurons and often spanning both hemifields). The distributions of receptive field sizes of MT and MST neurons were clearly segregated (Supplementary Fig. 1), and the percentage of neurons with receptive fields including ipsilateral regions was far higher in MST (56\%) than in MT (12\%).

We positioned the sample inside the MT or MST neuron's receptive field and chose four orthogonal sample directions with one of them matching the neuron's preferred direction. As a control, $70 \mathrm{MT}$ neurons were recorded with one of the two locations of the test stimuli, but not the sample location, inside the receptive field. $12 \mathrm{MT}$ and $30 \mathrm{MST}$ neurons were recorded with the sample and tests at the same location inside the receptive field.

Because the activity of LPFC neurons is highly task-dependent, we did not use the mapping task to characterize their response properties. Instead, we recorded while the monkey performed the delayed match-to-sample task immediately after isolating a neuron. It has been shown that most LPFC neurons have large receptive fields and show space-independent responses to visual motion ${ }^{10}$. We chose the sample position and directions to match the MT neuron's receptive field properties.

Data analysis. All analyses (unless otherwise indicated) were conducted in data recorded in correctly performed trials. Across all neurons, the mean number of correct trials recorded for each neuron was 140 . To display the firing rates of example neurons over time (Fig. $\mathbf{2 b}-\mathbf{g}$ ), we computed spike-density functions using a normal Gaussian kernel $(\sigma=40 \mathrm{~ms})$. Results obtained from both monkeys were qualitatively similar. Therefore, all neurons from both monkeys were pooled together for analysis. We analyzed neuronal activity during the sample and delay periods. One possible confounder in our paradigm is the fact that sensory neurons show residuals of the sensory response during the first few hundred milliseconds after stimulus offset. Two factors contribute to this phenomenon: first, a stimulus response takes a brief period of decay after stimulus offset while it returns to baseline firing rate ${ }^{31,32}$; second, in many neurons, sensory stimulation causes a brief period of neuronal adaptation (see Discussion). In order to 
avoid such confounders, all analyses of delay-period activity excluded data from the first $240 \mathrm{~ms}$ after the sample offset. Similar percentages of delay-selective neurons were obtained by excluding the first $480 \mathrm{~ms}$ instead of $240 \mathrm{~ms}$ of the delay period (Supplementary Fig. 2d). Thus, the presence of sustained activity in MST and LPFC, but not in MT, was not dependent on the precise criterion of time exclusion from the delay period.

Given that the duration of the delay period varied across trials between $1,200 \mathrm{~ms}$ and 2,000 ms, we only analyzed delay period activity until 1,200 ms after the sample offset. To assess whether each neuron encoded the sample direction over time, we tested for significant differences in firing rates between trials with different sample directions across time bins of $120 \mathrm{~ms}$ using a mixed between-within two-factor analysis of variance (ANOVA) with sample direction as a 'betweensubjects' factor and time bin as a 'within-subjects' factor. Each neuron was classified as direction-selective if it had a significant $(P<0.05)$ main effect of direction in at least one of two ANOVAs: one using time bins from the sample presentation period (sensory selectivity) and the other using time bins from the delay period (delay selectivity).

To determine the percentage of sensory-selective and delay-selective neurons that would be expected by chance, we randomly shuffled, for each neuron, the sample direction labels of all trials. We then performed the same two-factor ANOVA to obtain a surrogate percentage of sensory-selective and delay-selective neurons. The analysis was repeated 500 times to obtain 500 surrogate values of percentage of selective neurons. Percentages were considered significantly higher than chance if they were ranked within the top 95th percentile among all 500 surrogate values.

To test whether in some neurons, especially in area MT, the memorized direction could be represented very weakly in each individual time bin but more strongly in the average response across the entire delay, we conducted a one-factor ANOVA using the mean firing rate across each entire period (baseline, sample and delay). However, the percentages of neurons with a significant main effect of motion direction during the sample period (sensory selectivity) and delay period (delay selectivity) were similar to those obtained with the two-factor ANOVA (Supplementary Fig. 2c).

Receiver operating characteristics analysis. To quantify neurons' ability to discriminate between sample motion directions, we performed a ROC analysis. For each neuron, we computed the auROC to measure the separability of the distributions of firing rates between all possible pairs of sample directions. The auROC was computed across a sliding time window of $200 \mathrm{~ms}$ shifted by increments of $40 \mathrm{~ms}$. auROC values between 0 and 0.5 were rectified to their corresponding values in the range between 0.5 and 1 . Among all pairs of sample directions, the two directions for which the mean auROC across the sample and delay periods was highest were chosen as preferred and least-preferred directions. The auROC computed between these two sample directions was used to measure each neuron's direction discriminability and to compute population averages (Figs. 2 and $\mathbf{3}$ ).

To test whether auROC values were significantly higher than expected by chance, we performed a permutation test in which we shuffled preferred-sample and least-preferred-sample trial labels and computed the auROC between the shuffled trials. This procedure was repeated 500 times. An auROC was considered significant if it reached or exceeded the 99th percentile of the distribution of the 500 shuffled surrogates. This was performed through all steps of the sliding time window to detect periods of significant direction discriminability. These periods were further separated into those in which the unrectified auROC values were above and below 0.5 (Fig. 3a,c,e).

For each neuron, we added the duration of all significant bins to obtain the total duration of discriminability in each task period (Fig. 3i and Supplementary Fig. 3a,c); to obtain the maximum duration of consecutive discriminability in each task period, we identified the time segment with the maximum number of consecutive significant bins (Supplementary Fig. 3b,d). For each task period, the average of the 500 surrogate auROC values for each neuron was used to compute the mean auROC across neurons expected by chance (Fig. $\mathbf{3 h}$ ).

auROC in correct versus error trials. For each delay-selective MST and LPFC neuron, the aforementioned ROC analysis was repeated on error trials as well as on a randomly chosen downsampled set of correct trials that matched the number of error trials. We then computed the mean auROC across the delay period independently for correct and error trials (Fig. $\mathbf{5 c}, \mathbf{d}$ ), and computed the difference in auROC between them, $\triangle$ auROC $\left(\right.$ auROC $_{\text {correct }}-$ auROC $_{\text {incorrect; }}$; Fig. 5e). We tested whether the mean $\Delta$ auROC across neurons was significantly different from 0 using a one-sample $t$-test. We compared mean $\Delta$ auROC between MST and LPFC using a two-sample $t$-test.

Population decoding analysis. To estimate how strongly the activity of the population of recorded neurons in each area encoded the sample direction over time, we performed linear discriminant analysis (LDA). For each area, we generated a pseudo-population of neurons. This procedure is commonly used to estimate the coding ability of neuronal populations. It is important to note that because neurons were not recorded simultaneously, the resulting estimates of decoding accuracy are approximations of the coding ability of each population.

LDA was performed on the firing rates of neurons across a sliding window of $200 \mathrm{~ms}$ in steps of $40 \mathrm{~ms}$ using a leave-one-out cross-validation method to decode the sample direction. We excluded neurons with less than 30 trials per sample direction. The number of neurons used for the analysis from each area was randomly downsampled to match that of the area with the lowest number. Before training, we $z$-scored all firing rates and performed feature preprocessing on the training set of trials at each time window, consisting of an ANOVA to select neurons with a significant main effect of sample direction on the firing rates in that window.

For each brain area, we randomly selected 30 trials per condition from each neuron. Trial simultaneity among neurons was then randomly assigned between trials of the same condition. Decoding accuracy was computed as the percentage of trials for which the sample direction was correctly decoded. The above procedure was repeated 50 times, randomly reassigning trial simultaneity each time. At each time window, we then computed mean decoding accuracy and standard error across all repetitions (Fig. 4). To compute chance levels of decoding accuracy, we performed the above procedure after randomly shuffling sample direction labels between all trials. At each time window, significance of decoding accuracy was tested by comparing the distribution of decoding accuracy values with those expected by chance using a $t$-test (Bonferroni-corrected for multiple time-window comparisons). Decoding accuracy was compared between MST and LPFC with a $t$-test between the distributions of accuracy values of the two areas at each time window (Bonferroni-corrected for multiple time-window comparisons). A comparable analysis using support vector machine instead of LDA yielded similar results.

Choice probability. For each neuron, we computed choice probability as the auROC between the mean firing rates during the delay in correct and error trials, separately for trials with the sample in the preferred, least-preferred and intermediate directions (averaging across these latter directions). One-sample $t$-tests (one-tailed) were used to test whether mean choice probability across neurons in each area was significantly higher than 0.5 in trials with preferred, intermediate or least-preferred sample directions (Fig. 6c,d).

To test whether each neuron's choice probability was significantly higher or lower than expected by chance, the behavioral outcomes (correct or error) of all trials were randomly reassigned and a surrogate choice probability value was computed. We repeated this 500 times to obtain 500 surrogates. If the neuron's choice probability was in the top or bottom 2.5 percentile $(\alpha=0.05)$, it was considered significant. For each neuron with significant choice probability, we computed the time course of choice probability across a sliding window of $200 \mathrm{~ms}$ in steps of $40 \mathrm{~ms}$. At each time step, we then computed the mean and standard error of choice probability across these neurons (Supplementary Fig. 4).

To test whether the relationship between the memorized sample direction and the neurons' direction preference had a significant effect on choice probability, we performed a one-factor repeated-measures ANOVA with choice probability as the dependent variable and sample direction as a factor with three levels (sample in preferred, intermediate or least preferred directions), testing the simple main effect of sample direction on choice probability across neurons.

Local field potentials. Spectral analyses of LFP data were performed using multitaper methods in the Chronux Toolbox for Matlab (number of tapers $k=3$, time-bandwidth product TW $=2$, frequency range of $1-140 \mathrm{~Hz}$, see http://www. chronux.org/). We divided the LFP frequency spectrum into bands: theta $(\theta, 4-8 \mathrm{~Hz})$, alpha $(\alpha, 8-12 \mathrm{~Hz})$, beta $(\beta, 12-25 \mathrm{~Hz})$, low gamma $\left(\gamma_{\mathrm{L}}, 25-55 \mathrm{~Hz}\right)$ and high gamma $\left(\gamma_{\mathrm{H}}, 65-135 \mathrm{~Hz}\right)^{26}$. Increasing the high gamma range to $250 \mathrm{~Hz}$ did not change any of the results. Recording sites with less than 15 trials per sample direction were excluded from analysis. For each recording site, single trial power spectrograms were computed over the entire delay period (240-1,200 ms after sample offset). LFP power was normalized by dividing it by the mean power during the fixation period preceding the sample presentation. We excluded 
trials with mean normalized power in any band that exceeded 3 standard deviations of the power distribution across trials. These were rare, likely caused by occasional movements of the animal. Within each frequency band, ROC analysis was performed using the mean normalized power during the delay period in individual trials. The two sample directions yielding the highest auROC were selected as preferred and least preferred directions, and were used to measure the direction discriminability of LFP power (Fig. 7). To graphically display the time course of LFP power for the example LFP site in Figure 7a, the power spectrogram was computed across a sliding window of $240 \mathrm{~ms}$ in steps of $40 \mathrm{~ms}$.

For each band, we tested for significant discriminability of each LFP site by comparing the real auROC against 500 shuffled surrogate values (permutation test, $\alpha=0.05$; Fig. $7 \mathbf{b}$ ). To obtain the false positive rate of significant discriminability (i.e., the percentage of significant sites expected by chance) for each area and frequency band, we repeated the above analysis replacing the auROC of each site with a randomized surrogate, and computed the percentage of significant sites. We repeated this process 500 times and averaged the resulting surrogate percentages to obtain a mean percentage expected by chance (Fig. $\mathbf{7 b}$ and Supplementary Fig. 5a,b). For each brain area and frequency band, the percentage of sites with direction discriminability was considered significant if it exceeded the 95th percentile of the surrogate values. To compute the mean auROC among significant sites expected by chance, we averaged all surrogate auROC values for each site and computed the mean across all significant sites (Fig. 7c and Supplementary Fig. 5c,d).

Spike-field phase coherence. Spike-field phase coherence analysis was performed using multitaper methods in the Chronux toolbox and the same parameters described above. For each trial, phase coherence between the LFPs from an MT site and simultaneously recorded spikes from each LPFC neuron during the delay period was computed as a function of LFP frequency and then averaged across all trials (Fig. 8a,b). Spike-field pairs with less than 50 trials were excluded from analysis. We computed surrogates of coherence by randomly shuffling trial labels of LFP data while keeping the same labels for the spike data. This procedure was repeated to obtain 500 surrogates of coherence. The mean and 99th percentile of all surrogates as a function of LFP frequency was computed using a sliding $5-\mathrm{Hz}$ window in steps of $1 \mathrm{~Hz}$. Coherence was considered significant in frequencies reaching the 99th percentile of the surrogates' distribution (Fig. 8a,c). Each LPFC-MT pair was classified as significantly coherent if coherence reached significance in a range of at least $5 \mathrm{~Hz}$.

To correct for multiple comparisons resulting from testing significance using a sliding frequency window, we chose an alpha level that would yield a low incidence of false positives. We measured this incidence by repeating the significance test on surrogate values and computing the percentage of significant pairs. Using an alpha of 0.01 , the incidence of false positives was $0 \%$.

For pairs with significant coherence, we also performed phase coherence analysis using data from error trials. We computed the percentage of pairs with significant coherence as a function of frequency and then averaged the percentages across all frequencies within each frequency band independently for correct and error trials. We computed the percent reduction in the percentage of significant neurons as $100 \times(\mathrm{C}-\mathrm{E}) / \mathrm{C}$, in which $\mathrm{C}$ and $\mathrm{E}$ are the percentages of significant neurons in correct and error trials, respectively. We obtained similar results when repeating this procedure after downsampling the number of correct trials to match the number of error trials and equating the mean firing rates in correct and error trials by probabilistically removing spikes.

For each frequency band, we applied the Rayleigh test of uniformity to test whether the phases of coherence among all coherent pairs were non-uniformly distributed along the oscillatory LFP cycle, and measured their concentration with the concentration parameter kappa. 